Hydraulic Engineering Repository

Ein Service der Bundesanstalt für Wasserbau

Gris, Robie Bonilla

Sheath for Reducing Local Scour in Bridge Piers

Verfügbar unter / Available at:

https://hdl.handle.net/20.500.11970/100232

Vorgeschlagene Zitierweise / Suggested citation:

Gris, Robie Bonilla (2010): Sheath for Reducing Local Scour in Bridge Piers. In: Burns, Susan E.; Bhatia, Shobha K.; Avila, Catherine M. C.; Hunt, Beatrice E. (Hg.): Proceedings 5th International Conference on Scour and Erosion (ICSE-5), November 7-10, 2010, San Francisco, USA. Reston, Va.: American Society of Civil Engineers. S. 987-996. 


\title{
Sheath for Reducing Local Scour in Bridge Piers
}

\author{
Robie Bonilla Gris ${ }^{1}$, Member, ASCE \\ ${ }^{1}$ Professor (retired), Instituto Politécnico Nacional, México, Retorno de Chapuli- \\ nes \# 316, Frac. Club de Golf, Tequisquiapan, Qro., México, 76750, email: ro- \\ bie_bonilla@yahoo.com
}

\begin{abstract}
A sheath is presented which reduces the local scour around structures placed in a fluvial, lacustrine, estuarine, coastal or marine environment susceptible to scour. Such structures can be piers and abutments of bridges, columns, and other objects. The sheath has an especially designed rugosity which reduces the effect of the secondary flow that occurs in the border of attack of the structure. Additionally, the sheath can include complementary elements to give the ensemble a streamlined profile, enhancing the anti-scour performance. Also, it could be configured to rotate freely to align with a variable direction of flow, if needed. Preliminary experimental work indicates that the sheath could be a solution against local scour, the main cause of collapse of bridges. The sheath could be costeffective; could withstand any hydraulic conditions; its installation would not need heavy equipment; does not provoke alterations in the watercourse; can substitute environment-altering solutions; being underwater, does not interfere with the structure's aesthetics; could be used as a preventive or as a remedial measure.
\end{abstract}

\section{INTRODUCTION}

A problem frequently found in the case of structures and objects submerged in moving bodies of water is the local scour or erosion of material of the bottom in which they lean on, induced by the alteration of the flow caused by the presence of those structures and objects. This alteration consists of local increases of velocity and of the onset of secondary flows and of turbulent wakes with whirls or vortices. The local scour removes material of the bottom around the structures and objects and can put at risk their support, threatening their stability and its security and those of the corresponding superstructure, if any.

In spite of the technical development achieved through research (theoretical, experimental and with the help of numerical simulation and with field studies), carried out in institutions of many countries, and in spite of the interest of the official institutions in charge of the ground communications infrastructure and in spite of the enormous amounts of money invested in the search of solutions, scour is the main cause of the collapses of bridges, worldwide. $60 \%$ of those disasters is due to this phenomenon; because of that, nowadays it is still a factor of paramount importance in the design of these structures and an urgent technical 
challenge to solve (Jones, 2000; Khotyari, 2001; Parker, 1995; Briaud, 2005; Annandale, 2002).

In the United States, 17,000 bridges are considered in critical state with regard to scour (Briaud, 2005). The great number of bridges (about 600,000 in the United States) (Briaud, 2005) gives an indication of the problem dimension, and of the dramatic impact in the economy. The costs directly related to the bridge collapses are always very high. For example, $19 \%$ of the federal emergency funds of the United States used in the item of highways are used in the restoration of bridges. It should be added to the direct costs of a bridge collapse the indirect costs due to the serious affectations to roads and the dysfunctions in many activities; such costs can be even higher that the direct costs: the Federal Highway Administration estimates that these indirect costs can be five times the direct ones (Annandale, 2002). There is also a cost of prevention of those disasters.

The collapses of bridges, above all, are an important risk for the public security: human lives have been lost in those disasters.

Other examples of structures susceptible to damage due to local scour are piles, columns, supports of structures or equipment or machines, and similar structures, leaning or anchored in the bed or bottom or in the riverbanks of a ravine, a body of water or an artificial conduct or in a fluvial, lacustrine, estuarine, coastal or marine environment.

\section{THE LOCAL SCOUR}

The collapse of a bridge due to local scour generally begins with the loss of support of one or more piers. One of the abutments can also fail.

Local scour is produced by a complex turbulent flow that is mainly the effect of two independent, well-known mechanisms. A brief explanation of those two different causes of the local scour follows.

First mechanism: The horseshoe vortex. Figure 1 shows a submerged structure or object, leaning on an erodible bottom. The flow 11 that collides against the border or attack area of the structure $\mathbf{1 0}$ (the area that directly faces the current or flow) is deflected downward by the vertical gradient of the stagnation velocities, generating this way the secondary flow 12 , which travels vertically, in close contact with the front of attack of the structure. The secondary flow powers the socalled horseshoe vortex 13. This vortex surrounds the submerged structure or object, in contact with the bottom, and spreads downstream, transporting sediment detached by the secondary flow and also removing material from the bottom around the structure; that material is then carried away by the current, giving place to the scour hole.

This first mechanism is the main responsible for the local scour. 


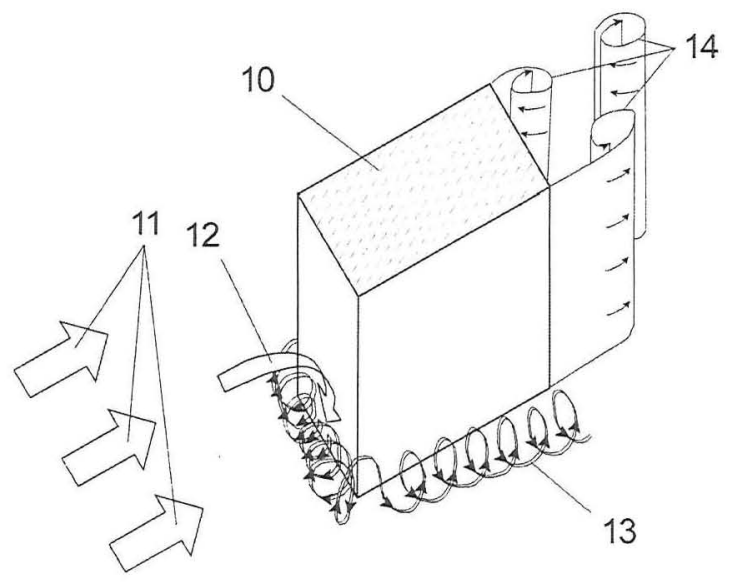

FIG. 1. Local scour mechanisms

Second mechanism: wake vortices $\mathbf{1 4}$ (Fig. 1). The flow that surrounds the submerged structure or object produces vertical vortices called wake vortices. These vortices tend to appear periodically and alternately from one side and the other of the structure and are carried away by the flow. As small tornadoes, they remove particles from the bottom and put them to movement; the flow transports them and so completes the second local scour mechanism. The wake vortices belong to the turbulent wake caused by the presence of the structure inside the flow.

If the depth of the scour hole, basically due to the combined effect of the two described mechanisms, surpasses certain critical magnitude, the support of the structure is reduced and its security is at risk.

\section{THE STATE-OF-THE-ART}

The current state-of-the-art consists on facing the problem with the following main types of measures intended to reduce the scour effects around the submerged structure:

1. The protection of the bed or bottom near the submerged structure, using one or more of the following resources: rock, monolithic precast and cast-in-place concrete structures, crushed concrete, riprap, mattresses or mats of several types constituted by such heavy elements as bags made of plastic meshes or geotextiles containing concrete or stones, layers of stones retained and separated by geotextiles, gabions, concrete blocks tied to each other by steel cables, and other resources. 
2. The construction on or near the submerged structure of some appendixes or elements, whose shape helps to deviate the flow away from the structure, or whose position and shape tend to move the scour away from the structure, such as spurs, flow deflectors, semicircular or triangular endnoses, protection slabs, collars, sacrifice piles and other elements.

3. The construction or setting of the structure foundation to considerable depths, greater than the scour depths estimated by means of the available calculation formulae. The reason of this measure is that such formulae don't give very reliable results; their error margins are generally big. Also, their application is limited because they don't consider the cases of complex flows, like those that include surf and variable currents; furthermore, they don't take into account complex geotechnical characteristics of the bottom and are only applied to simple shapes of piers.

From the functional point of view, the described measures, which constitute the main body of the current state-of-the-art, have as objective to reinforce the scour-susceptible bed or bottom, or to move away the phenomenon of scour away from the structure or object to protect, or set the structure to a depth that responds more to a collapse fear than to a rational, engineering decision. That is, the state-of-the-art attempts, at great cost and without much success, to reduce the effects of local scour, without directly attacking the cause of the phenomenon itself. The statistics of vulnerability of the submerged structures to the action of local scour show that the protection obtained nowadays is faulty and that there is an urgent need of better solutions.

Only some relatively recent developments are based on the control of the hydrodynamics responsible for the mechanisms that produce the local scour. The FHWA Sterling Jones Hydraulics Research Laboratory (2007) has a current research project which considers using streamlining of the shape of a bridge pier and also roughness elements on the surface of the pier. Khwairakpam and Mazumdar (2009) report about some flow-altering techniques, using splitter plates or threaded piles (Day, S.; Sumer, B. M. and Fredsøe, J., 2006) and pier slots and collars (Kumar, V., Ranga Raju, K. G. and Vittal, N., 1999).

\section{DESCRIPTION OF THE SHEATH}

The patented sheath presented here (Bonilla Gris, R., 2009) faces the problem of local scour attacking its origin itself, modifying conveniently the flow near the submerged structure or object, in order to reduce the intensity and the effect of the mechanisms of local scour.

The sheath (Fig. 2) is to be placed around the exterior surface of the structures and objects submerged in a current or flow. Those structures and objects can have any shape and can be made of any material. 


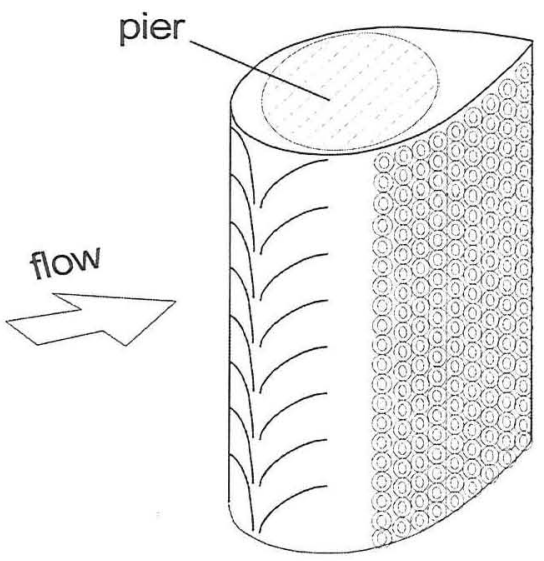

FIG. 2. The pier sheath

The sheath has the following basic features:

(a) A specially designed artificial directional rugosity in the front of attack, whose characteristics will be described later on.

This artificial rugosity has a decisive influence on the downward secondary flow through the creation of systems of inclined vortices which are carried away by the current without arriving to the bottom, resulting in a damping of the vertical momentum of the secondary flow.

(b) A specially designed non-directional rugosity in the rest of the external surface, which gives the sheath a "golf ball" effect; that is, transforms the laminar boundary layer to turbulent relocating the flow separation line downstream of the position corresponding to a smooth pier. This way, the wake reduces its strength and so do the wake vortices.

The current local scour state-of-the-art, in general, doesn't take into consideration the characteristics of the surface of the submerged structures; it is customary to give it a smooth finish, when the structure is made in a form (as in the most frequent case, of concrete structures), and to keep unchanged the natural rugosity of the construction material, in other cases.

(c) Optionally, an efficient hydrodynamic, streamlined profile that reduces the area of the front of attack -then the secondary flow and the horseshoe vortex momenta-- and produces a minimum turbulent wake. In this option, the nondirectional rugosity is excluded. 
In the current state-of-the-art, it is not usual to give the submerged structures and objects a very convenient hydrodynamic (streamlined) profile, due to high construction costs because forms of complex geometry are needed, when the structures or the objects are built of reinforced concrete or another formed material; also, there are structural and damage susceptibility issues.

(d) Also, optionally, the streamlined ensemble could be so made as to rotate freely around the structure, to align itself automatically with a variable direction of flow.

The sheath would be put on the pier so that its directional rugosity is located in the border or area of attack of the submerged structure. In the case of structures located in flows whose direction is variable, as in estuarine, coastal and marine environments or in rivers with tide influence, the directional rugosity would face the different directions, or would be put in the whole perimeter; also, the sheath could be made so as to have the hydrodynamic profile and to rotate freely to align automatically with a variable direction of flow.

The sheath is conceptually made of the cover (Fig. 2) that contains the two types of artificial rugosity; optionally, the sheath may include one or more intermediate components fixed to the submerged structure. Those intermediate components would give the ensemble the streamlined profile and hold the cover in place.

The rugous cover could be formed out, for example, of a flexible, water- and weather-proof material, as well as resistant to the particular characteristics of the flowing liquid (it must be resistant to brackish or salt water found in the estuarine, coastal and marine areas). It also must have appropriate mechanical characteristics in order to be fastened to the intermediate components, or directly to the submerged structure or object, if no intermediate elements are present, and to remain this way. The cover would resist cutting, tearing and bumping due to floating matter. It must also fulfill the environment protection standards.

The intermediate component or components would be manufactured from a material which can be conveniently formed and that keeps its shape and dimensions along the work life of the sheath. It would also be capable of being held by means of a simple procedure to the structure or object to be protected; for example, it can be made up of two or more complementary parts that can be united to each other in a simple way. The intermediate component would also be capable of holding fast the cover.

The directional rugosity is constituted by linear rugous elements in the shape of stripes distributed in an essentially symmetrical way with regard to the vertical axis of the border of attack of the structure (Fig. 2). The non-directional rugosity consists of a multiplicity of individual rugous elements.

Due to the fact that the vertical gradient of the stagnation velocities increases with the water depth, making the secondary flow become gradually swifter as it approaches the bed, the sheath should be fixed on the pier so as to sit on the bed. Also, the most important stretch of the vertical dimension of the sheath being the lower part, the sheath's height could be less than the design water depth. 
The parameters of the hydrodynamic profile would depend on the pier shape and size and on the Froude Number range of the watercourse or of the water environment velocities. As for floating debris, the hydrodynamic profile of the sheath, having the same maximum width as the pier (with a difference of less than $10 \mathrm{~cm}$ ) and a smaller area of attack, it seems that would retain less debris than the pier itself; the inclined and curved stripes of the rugosity would also help.

\section{EXPERIMENTAL RESULTS}

The advantageous results of the application of the artificial rugosity of the sheath in the reduction of local scour, have been proven in 2 preliminary experimental studies in a Hydraulics laboratory, using scale physical models with the characteristics indicated in the following abbreviated description; in that description the following dimensionless hydrodynamic parameters are used:

$$
F=\frac{U}{\sqrt{g d}}
$$

(Froude Number), where $U$ is the mean velocity of the flow, $g$ is the acceleration due to gravity and $d$ is the flow depth.

$$
R=\frac{U D}{v}
$$

(Reynolds Number), where $U$ is the mean velocity of the flow, $D$ is the diameter of the cylindrical pier and $v$ is the coefficient of kinematic viscosity of the liquid.

In the first study, a channel of rectangular section $0.56 \mathrm{~m}$ wide, a flow of 21.6 1/s were used and as bottom material, ground Bakelite with an initial transport velocity of $0.14 \mathrm{~m} / \mathrm{s}$. In the experiment, cylindrical piers with a diameter of 0.07 $\mathrm{m}$ were used and the flow depth was $0.27 \mathrm{~m}$. The Froude Number was 0.09 and that of Reynolds, $8.8 \times 10^{3}$.

The second study was carried out in a channel of rectangular section $1.50 \mathrm{~m}$ wide, with a flow that varied between 49.5 and $59.4 \mathrm{l} / \mathrm{s}$; the material of the bottom was sand and its initial transport velocity was $0.22 \mathrm{~m} / \mathrm{s}$. Cylindrical piers $0.10 \mathrm{~m}$ in diameter were used; the flow depth varied from $0.12 \mathrm{~m}$ to $0.20 \mathrm{~m}$. The values of the Froude Number varied between 0.14 and 0.27 and the values of the Reynolds Number, between $1.7 \times 10^{4}$ and $2.7 \times 10^{4}$.

Both series of experiments were done in clear-water sediment conditions.

The tests were done to assess the scour-reducing performance of the artificial roughness created on a cylindrical pier, relative to a cylindrical smooth pier with the same diameter in the same hydraulic and sediment conditions. Because of 
this restraint, several parameters were left out of the picture as variables: streambed and sediment characteristics, depth and angle of attack of flow, shape and relative obstruction of the pier. The duration of every couple of tests (smooth pier, rugous pier) was the same; they were stopped when the smooth pier scour depth was no longer time-dependent.

As already noted, no streamlined profile was added to the model piers.

In every experiment, with a variety of rugosities, the maximum scour depth and the total scour volume were calculated.

The results of both laboratory studies showed a significant reduction of the depth and volume of the scour hole in piers with the artificial rugosity, in comparison with the values corresponding to a smooth pier under the same hydrodynamic and sediment conditions (Fig. 3). The reduction in scour depth ranged from $20 \%$ to $30 \%$. A scour hole topography different from that of the wellknown smooth pier scour hole was found.

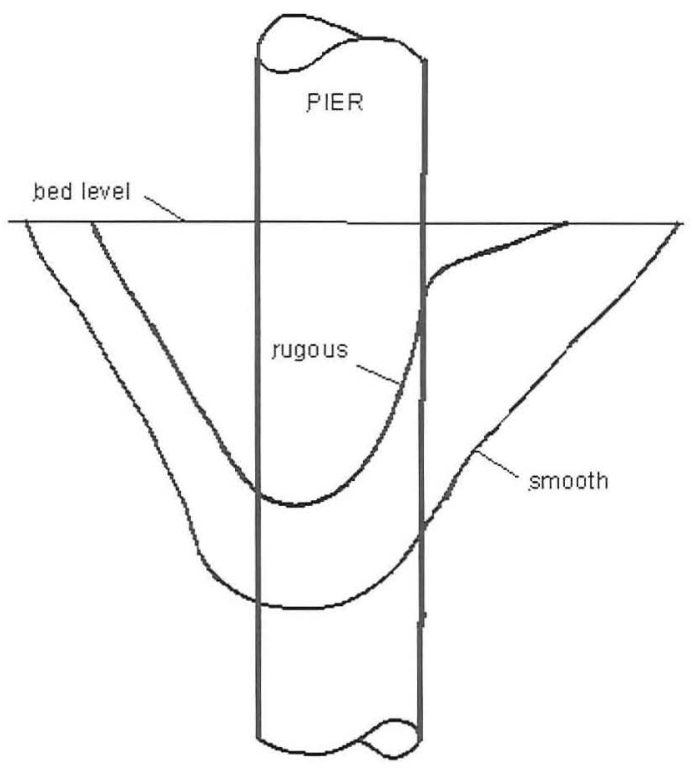

FIG. 3. Scour holes for rugous and smooth piers (typical) 


\section{CONCLUSIONS AND RECOMMENDATIONS}

1. In comparison with the common measures and solutions provided by the state-of-the-art, the sheath, besides being efficient in the reduction of the local scour, has the following advantages:

(a) It can be applied, as a preventive measure against local scour, to recently built or placed submerged structures and objects; also, as an attenuation or remediation measure, can be applied to others that already run risks or have problems due to this phenomenon.

(b) It could be cost-effective, since the application of the sheath would not require considerable additional work, neither additional times of construction, or heavy equipment.

(c) It can resist any hydrodynamic conditions of velocity and turbulence.

(d) The optional streamlined profile of the sheath is independent of the profile of the submerged structure, so that the sheath can be applied to any shape of horizontal section of such structure.

(e) It doesn't increase the underwater profile of the structure, and then it does not produce parasite scour effects downstream of the structure, which could do harm.

(f) It doesn't interfere with the aesthetics of the structure, since the sheath can be applied under the water level.

(g) The technology is simple, of easy transfer and application.

(h) The technology can substitute environment-altering solutions.

2. The addition of artificial rugosity to the surface of a pier as a local scour countermeasure sets a new paradigm -the state-of-the-art of local scour depth assessment (formulae, parameters, criteria) assumes beforehand that the pier or structure is smooth--. In fact, the experimental work mentioned shows that the smooth pier is the worst performer in respect to local scour. This means that a new parameter representing rugosity should be included in the current formulae.

3. The experimental results presented here were obtained in cylindrical piers; the streamlined sheath, having a smaller front of attack, should generate a weaker secondary flow and horseshoe vortex; then, better anti-scour performance. This conclusion can be applied also to the downstream end of the sheath; the wake vortices produced will be less strong. 
4. As mentioned, the hydrodynamics of the lab tests were observed without high-speed instrumentation; then, the flow near the rugosity must be studied with more precise methods to fully assess and optimize the artificial rugosity effect.

5. The results presented here should be confirmed by extensive laboratory tests and also by tests in real structures.

Some pending studies are:

(a) Refined rugosity characteristics for smallest scour depth (for different Reynolds Number ranges);

(b) Best hydrodynamic profiles for different ranges of flow conditions;

(c) Needed vertical extent of the sheath (probably, not through the entire water depth).

\section{REFERENCES}

Annandale, G. W., Melville, B., Chiew, Y. (2002). "Scour Case Studies". Mitteilungsblatt der Bundesanstalt für Wasserbau, Nr. 85 (Information Newsletter of the Federal Institute of Rivers and Channels Engineering and Research, Germany, (No. 85)).

Bonilla, R., U. S. Patent No. 7628569 , PCT filed July 12, 2005.

Briaud, J. L. (2005). "SRICOS Sheds Light on Bridge Scour Problems". Texas Transportation Researcher, Texas Transportation Institute, (Vol. 34, No. 2).

FHWA Sterling Jones Hydraulics Research Laboratory, Current Research

Project: "Pier Scour Countermeasures Using Fluidic Devices". (2007).

Available at:

http://www. fhwa.dot.gov/engineering/hydraulics/research/summary.cfm

Hamill, L. (1999). Bridge Hydraulics, E\&F Spon, London and New York.

Jones, J. S. (2000). "Hydraulics Testing of Wilson Bridge Designs". Public Roads, Federal Highway Administration, U. S. Department of Transportation, (March/April 2000, Vol. 63 No. 5).

Khotyari, U. C.; Ranga R. K. G. (2001). "Scour Around Spur Dikes and Bridge Abutments". Journal of Hydraulic Research, (Vol. 39, 2001, No. 4).

Khwairakpam, P.; Mazumdar, A. "Local Scour Around Hydraulic Structures". International Journal of Recent Trends in Engineering, ACEEE, (Vol. 1, No. 6, May 2009).

Parker, G., Voigt, R. (1995). "Countermeasures to Protect Bridge Piers from Scour". National Cooperative Highway Research Program, (NCHRP Project 24$7(2))$. 